\title{
Bioinformatics analysis and identification of circular RNAs promoting the osteogenic differentiation of human bone marrow mesenchymal stem cells on titanium treated by surface mechanical attrition
}

Shanshan Zhu ${ }^{1}$, Yuhe Zhu ${ }^{1}$, Zhenbo Wang ${ }^{2}$, Chen Liang ${ }^{2}$, Nan-Jue Cao ${ }^{3}$, Ming Yan ${ }^{1}$, Fei Gao ${ }^{4}$, Jie Liu ${ }^{\text {Corresp., }}$, , Wei Wang ${ }^{\text {Corresp. } 1}$

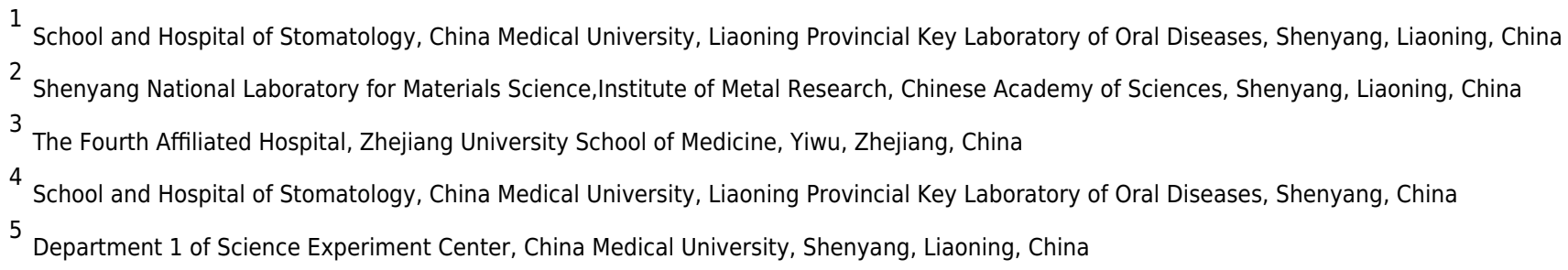

Background. To analyse and identify the circular RNAs (circRNAs) involved in promoting the osteogenic differentiation of human bone mesenchymal stem cells (hBMSCs) on titanium by surface mechanical attrition treatment (SMAT).Methods. The experimental material was SMAT titanium and the control material was annealed titanium. Cell Counting Kits-8 (CCK-8) was used to detect the proliferation of hBMSCs, and alkaline phosphatase (ALP) activity and alizarin red staining were used to detect the osteogenic differentiation of hBMSCs on the sample surfaces. The bioinformatics prediction software miwalk3.0 was used to construct competing endogenous RNA (ceRNA) networks by predicting circRNAs with osteogenesis-related messenger RNAs (mRNAs) and microRNAs (miRNAs). The circRNAs located at the key positions in the networks were selected and analyzed by quantitative real-time PCR (QRT-PCR).Results. Compared with annealed titanium, SMAT titanium could promote the proliferation and osteogenic differentiation of hBMSCs. The total number of predicted circRNAs was 51. Among these, 30 circRNAs and 8 miRNAs constituted 6 ceRNA networks.Circ-LTBP2 was selected for verification. QRT-PCR results showed that the expression levels of hsa-circ-0032599, hsa-circ-0032600 and hsacirc-0032601 were upregulated in the experimental group compared with those in the control group; the differential expression of hsa-circ-0032600 was the most obvious and statistically significant, with a fold change $(\mathrm{FC})=4.25 \pm 1.60, p$-values $(p)<0.05$. 


\section{Bioinformatics analysis and identification of circular RNAs}

2 promoting the osteogenic differentiation of human bone

3 marrow mesenchymal stem cells on titanium treated by

4 surface mechanical attrition

5 Shanshan Zhu ${ }^{1, *}$, Yuhe Zhu ${ }^{1,}{ }^{*}$, Zhenbo Wang ${ }^{2}$, Chen Liang ${ }^{2}$, Nanjue $\mathrm{CaO}^{3}$, Ming Yan ${ }^{1}$, Fei Gao ${ }^{1}$, Jie Liu ${ }^{4}$, Wei

6 Wang $^{1}$

$7 \quad{ }^{1}$ School and Hospital of Stomatology, China Medical University, Liaoning Provincial Key Laboratory of

8 Oral Diseases, Shenyang, Liaoning, China

9 2Shenyang National Laboratory for Materials Science, Institute of Metal Research, Chinese Academy of

10 Sciences, Shenyang, Liaoning, China

$11{ }^{3}$ The Fourth Affiliated Hospital, Zhejiang University School of Medicine, Yiwu, Zhejiang, China

$12{ }^{4}$ Department 1 of Science Experiment Center, China Medical University, Shenyang, Liaoning, China

13

$14{ }^{*}$ Authors contributed equally to this work

15 Corresponding Authors:

16 Wei Wang ${ }^{1}$, Jie Liư

$17{ }^{1} 117$ Nanjing North Street, Heping District, Shenyang, Liaoning, 110002, China

18 Email address: wwang75@cmu.edu.cn

$19{ }^{4} 77$ Puhe Road, Shenbei New District, Shenyang, Liaoning, 110013, China

20 Email address: lj6152003@163.com 


\section{Abstract}

22 Background. To analyse and identify the circular RNAs (circRNAs) involved in promoting the

23 osteogenic differentiation of human bone mesenchymal stem cells (hBMSCs) on titanium by 24 surface mechanical attrition treatment (SMAT).

25 Methods. The experimental material was SMAT titanium and the control material was annealed 26 titanium. Cell Counting Kits-8 (CCK-8) was used to detect the proliferation of hBMSCs, and 27 alkaline phosphatase (ALP) activity and alizarin red staining were used to detect the osteogenic 28 differentiation of hBMSCs on the sample surfaces. The bioinformatics prediction software 29 miwalk3.0 was used to construct competing endogenous RNA (ceRNA) networks by predicting 30 circRNAs with osteogenesis-related messenger RNAs (mRNAs) and microRNAs (miRNAs).

31 The circRNAs located at the key positions in the networks were selected and analyzed by 32 quantitative real-time PCR (QRT-PCR).

33 Results. Compared with annealed titanium, SMAT titanium could promote the proliferation and 34 osteogenic differentiation of hBMSCs. The total number of predicted circRNAs was 51. Among 35 these, 30 circRNAs and 8 miRNAs constituted 6 ceRNA networks. Circ-LTBP2 was selected for 36 verification. QRT-PCR results showed that the expression levels of hsa-circ-0032599, hsa-circ-

370032600 and hsa-circ-0032601 were upregulated in the experimental group compared with those 38 in the control group; the differential expression of hsa-circ-0032600 was the most obvious and 39 statistically significant, with a fold change $(\mathrm{FC})=4.25 \pm 1.60, p$-values $(p)<0.05$. 
40 Conclusions. Compared with annealed titanium, SMAT titanium can promote the osteogenic 41 differentiation of hBMSCs through circRNAs.

42 Key words: surface mechanical attrition treatment, human bone marrow mesenchymal ste $\mathrm{m}$ cells, biological information prediction, circRNA, osteogenic differentiation

\section{Introduction}

Oral implant technology can effectively repair dentition defects. Compared with traditional repair methods, oral implants have more beautiful shape, have stronger retention, result in higher chewing efficiency and lower foreign body sensation, and can delay the absorption of alveolar bone (Camargo WA et al., 2017). At present, dental implants are mainly made of pure titanium and its alloys because titanium has a low density, low modulus, high strength, and excellent biocompatibility and corrosion resistance (Song P et al., 2019; Duan M et al., 2019; Zhou W et al., 2019). However, the corrosion resistance of titanium after implantation in the human body is reduced, resulting in "stress shielding" that causes fibrous binding rather than osseointegration and affecting the stability of the implants (Sven H et al., 2018; Ito T et al., 2018; Minko D, Belyavin K, Sheleg V, 2017). Previous works showed that surface mechanical attrition treatment (SMAT) technology might be applied to solve this problem by improving osseointegration ability on Ti surface (Lai M et al., 2012). In addition, SMAT metals have the advantages of high strength (T.H. Fang et al., 2011), decreased surface alloying temperature/time due to high diffusion rate (Z.B. Wang, K. Lu, 2017) and high chemical reactivity (W.P. Tong et al., 2003), and their wear resistance and fatigue resistance are also significantly improved (Z.B. Wang et al., 2003; Y.B. Lei et al., 2019).

Human bone marrow mesenchymal stem cells (HBMSCs) are easy to acquire, isolate, culture and purify. They have strong proliferation and differentiation potential. After many passages, they still have the characteristics of stem cells; they function in haematopoietic support 
65 and can secrete a variety of growth factors. They also have an immunoregulatory function and 66 show low immunological rejection characteristics (Son J W et al., 2018; Miranda H C et al., 67 2012).

Circular RNA is double-stranded non-coding RNA that is bound by a covalent bond into a

69 closed loop structure without a 5' cap and 3' poly tail and is not easily degraded by exonucleases. It is more stable than linear mRNA, and its length is between $200 \mathrm{bp}$ and $2000 \mathrm{bp}$ (Zhao R et al., 2019; Ji H N et al., 2018); it is highly conserved, and it is found in eukaryotes, mostly within the cytoplasm. A total of $16 \%$ of circRNAs are derived from coding genes, while $85 \%$ are derived from exon rings. CircRNAs have specific reverse cleavage sites, and multiple circRNAs can be produced at the same locus; this mainly occurs at the post-transcriptional level, and most transcripts are non-encoding (Liu J et al., 2017; Zhao Z et al., 2018; Zhang M, Xin Y, 2018). CircRNAs show specificity in terms of timing, organization, and disease specificity. They accumulate in the nerve tissue during ageing and can be secreted from the outside of the cell to form a ring in the membrane. The main types of circRNAs include exon circRNAs, exon-intron circRNAs, intron circRNAs, antisense circRNAs, intergenic circRNAs, and sensory-overlap circRNAs. The main functions of circRNAs are as follows: to function as ceRNA molecules (as miRNA molecular "sponges") to bind to miRNA response elements (MREs) to reduce the effects of miRNA on gene expression; to regulate classical RNA splicing so that classical RNA is inhibited; to regulate parental gene transcription (Kulcheski F R, Christoff A P, Margis R., 2016; Cortéslópez M et al., 2018); to bind to RNA-binding proteins to regulate other RNAs; to be translated into proteins; to induce pseudogene expression after reverse transcription (Li S, Han L, 2019).

A large number of studies in recent years have shown that circRNA can regulate stem cell osteogenic differentiation. Zhang et al. (Mengjun Z, Lingfei J, Yunfei Z, 2018) performed microarray analysis to determine the expression profile of circRNAs during osteoblast differentiation. The results indicated that the functional annotation of differentially expressed circRNAs was associated with osteogenic differentiation. The researchers then constructed a 
92 circRNA-miRNA network, and network analysis indicated that some circRNAs were associated

93 with miRNAs with osteogenic effects. In addition, the researchers verified the expression of a

94 central miRNA, miR-199b-5p, and its associated circ RNA circIGSF11. The results showed that

95 silencing circIGSF11 can promote osteoblast differentiation; Zheng et al. (Zheng Y et al., 2017)

96 induced periodontal ligament stem cells (PDLSCs), which were analysed by circRNA

97 sequencing, qRT-PCR, differential expression analysis and gene ontology (GO) analysis. The

98 target mRNAs regulated by the differentially expressed circRNAs were enriched in cell matrix

99 formation and osteogenic differentiation.

100 This study intends to use biological information prediction to analyse the interactions

101 between miRNAs, circRNAs, and circRNA-miRNA-mRNA that interact with osteogenic

102 mRNAs. It also seeks to investigate whether the SMAT titanium material can promote hBMSC

103 osteogenic differentiation by affecting circRNA and provide a theoretical basis for the

104 application of SMAT in oral clinic.

105

106 Materials \& Methods

107 1. Preparation of titanium sheets

108 Experimental group: Pure titanium plates in medical grade, in a cylindrical shape with a

109 diameter of $60 \mathrm{~mm}$ and a thickness of $5 \mathrm{~mm}$, were studied in this work. We performed

110 ultrasonic-assisted SMAT on the plate samples by using a SNC-2 machine (New Nano-crystal

111 Technology Co., Ltd., China) at a frequency of $20000 \mathrm{~Hz}$. During SMAT, hardened steel balls

112 with a diameter of $6 \mathrm{~mm}$ were driven by the system and repeatedly impacted onto the sample

113 surface for $30 \mathrm{~min}$, so that a gradient nanostructured surface layer was formed. The SMAT plates

114 were electrically spark-cut into discs with a diameter of $11 \mathrm{~mm}$ and a thicknesses of $2.5 \mathrm{~mm}$, as

115 the samples in the experimental group. 
116 Controlled group: An annealing treatment was carried out at $680{ }^{\circ} \mathrm{C}$ for 2 hours on the

117 SMAT samples to prepare the samples in the controlled group. By doing this, the surface

118 morphologies of the experimental and controlled samples were similar, while the grains in the

119 surface layer of the controlled samples became coarse.

120 Samples in both the experimental and controlled groups were sequentially cleaned in

121 acetone, 36\%-38\% dilute hydrochloric acid, absolute ethanol and distilled water, and then

122 ultrasonically washed for 20 minutes, dried and autoclaved prior to use.

123 Transmission electron microscope (TEM; JEOL, Japan) was used to observe the surface

124 structure of the experimental materials so as to determine whether it has gradient nanostructures.

125 2. Culture of hBMSCs

126 HBMSCs were purchased from Beijing Yuhengfeng Technology Co., Ltd. We resuscitated

127 the 10th generation of hBMSCs and placed them in $\alpha$-modified Eagle medium ( $\alpha$ MEM, HyClone)

128 containing 15\% foetal bovine serum (FBS, HyClone) and $100 \mathrm{U} / \mathrm{ml}$ double antibody (HyClone),

129 and the cells were cultured at $37^{\circ} \mathrm{C}$ in a $5 \% \mathrm{CO}_{2}$ cell culture incubator. The cells were in the

130 logarithmic phase of growth during all experiments.

131 3. CCK-8 method for determining the cell proliferation curve

132 The 14th generation hBMSCs showing good growth were inoculated at $4 \times 10^{4} / \mathrm{cm}^{2}$ on

133 SMAT titanium and annealed titanium, which were placed in 24-well plates. Each group of

134 materials was placed in 3 wells. Each well was filled with $1 \mathrm{ml}$ of basic medium. A total of 100

$135 \mu$ of CCK-8 (KGI Biotechnology Co., Ltd., China) was added to each well on day 1, 3, 5, and 7.

136 We incubated the plates for 2 hours at $37^{\circ} \mathrm{C}$. Then, we pipetted $110 \mu \mathrm{l}$ of reaction solution per

137 well into a 96-well plate. A microplate reader (Tecan, Switzerland) was used to detect the optical

138 density (OD) value of each well at a wavelength of $450 \mathrm{~nm}$. The proliferation curve of the 14th

139 generation of hBMSCs was generated with time as the horizontal axis and the average OD as the

140 vertical axis. 
141

142

143

144

145

146

147

148

149

150

151

152

153

154

155

156

157

158

159

160

161

162

163

164

165

\section{ALP activity detection}

We inoculated 14th generation hBMSCs showing good growth at $4 \times 10^{4} / \mathrm{cm}^{2}$ on SMAT titanium and annealed titanium, which were placed in 24-well plates. Each group of materials was placed in 3 wells, and each well was supplemented with $1 \mathrm{ml}$ basic culture solution. When the cell fusion degree was $80 \%-90 \%$, we added osteogenic induction medium (15\% FBS, $1 \%$ streptomycin mixture, $10^{-7} \mathrm{M}$ dexamethasone, $10^{-2} \beta$-glycerophosphate disodium salt, $50 \mu \mathrm{g} / \mathrm{ml}$ Vitamin C, aMEM) and cultured the cells in a $37^{\circ} \mathrm{C}$ incubator. After 3, 5, 7, and 14 days of culture, we digested the cells of each group with trypsin (Solarbio). We lysed the cells with $1 \%$ Triton X-100 (Solarbio) and repeatedly thawed them until they were disrupted, and then we collected the supernatant. The ALP activity of each group of cells was determined and calculated according to the instructions in the ALP activity test kit (Institute of Bioengineering, Nanjing, China) and Bradfords (BCA) kit (Institute of Bioengineering, Nanjing, China).

\section{Alizarin red staining}

The 14th generation hBMSCs showing good growth were inoculated at $4 \times 10^{4} / \mathrm{cm}^{2}$ on SMAT titanium and annealed titanium, which were placed in 24-well plates. Each group of materials was plated in 3 wells supplemented with $1 \mathrm{ml}$ basic culture solution. When the cell fusion degree was $80 \%-90 \%$, we added osteogenic induction medium and cultured the cells in a constant temperature incubator. After 7, 14, and 21 days of culture, we fixed the cells in $95 \%$ ethanol for 1 hour and stained them with 1\% Alizarin red (Sigma) for 2 hours.

\section{Biological information prediction}

(1) Prediction and screening of osteogenesis-related miRNAs

The osteogenesis-related literature from 2015 to 2019 was used to select 20 osteogenesisassociated mRNAs. Twelve mRNAs associated with the transforming growth factor- $\beta$ (TGF- $\beta$ )/ drosophila mothers against decapentaplegic protein (Smad), mitogen-activated protein kinase (MAPK)/ extracellular signal-regulated kinase (ERK), Wnt, and Notch signalling pathways were 
166 analysed and screened in combination with Kyoto Encyclopaedia of Genes and Genomes

167 (KEGG) signalling pathway analysis. MiRNAs 3.0 software was used to screen for miRNAs

168 capable of targeting these candidate mRNAs. The underlying screening principle involved the

169 identification of miRNAs that were located in the seed area as predicted by TargetScan, miRDB

170 and miwalk3.0.

171 (2) The prediction and screening of osteogenesis-related circRNAs

172 The formation of circRNA is based on exon cyclization. Miwalk3.0 software was used to

173 screen the complementary sequence of the coding sequence (CDS) region of the obtained

174 miRNA, and the mRNA sequence of the seed region was used as the source transcript for the

175 circRNA. The exon of each gene was searched based on the complementary region, and the

176 circRNA including the exon was screened. The length of the circRNAs selected for the

177 experiment were 200-2000 bp, and each was detected in more than 2 samples from the circBase.

178 (3) Predictive analysis of ceRNA networks of osteogenesis-related circRNAs

179 The mRNA-miRNA-circRNA interactions were analysed to determine the frequency

180 distribution, and network analysis was performed by using Cytoscape software to identify the

181 core molecules and construct the ceRNA networks. The interactions between them were

182 analysed, and the selected circRNAs located at key locations in the networks were used for qRT-

183 PCR verification.

184 7. QRT-PCR detection of the differential expression of circRNAs during the osteogenic 185 differentiation of hBMSCs on the two groups of titanium plates

186 The 14th generation hBMSCs showing good growth were inoculated at $4 \times 10^{5} / \mathrm{cm}^{2}$ on

187 SMAT titanium and annealed titanium in 6-well plates. We used 3 wells for each set of materials, 188 and each well was filled with $3 \mathrm{ml}$ of basic culture solution. When the cell fusion degree was

$18980 \%-90 \%$, we added osteogenic induction medium and cultured the cells in a constant

190 temperature incubator. Once the cells had been osteogenically induced for 3, 5, 14, 21 days, two 
191 sets of total RNA were separately extracted with TRIzol (Gibco), and the purity and amount of

192 the collected RNA were determined by a Nanodrop 2000 microultraviolet analyser (Thermo

193 Company, USA). CDNA was synthesized using the PrimeScript RT Master Mix (Takara, Japan).

194 Shengong Biological Engineering Co., Ltd. (Shanghai, China) designed specific primers for the

195 circRNAs. QRT-PCR was performed using TB Green Premix EX TaqTM II (Takara, Japan), and

196 PCR-specific amplification was conducted with a Prism 7500 real-time PCR instrument (ABI

197 Company, USA). The expression of circRNAs was determined based on the threshold cycle (Ct),

198 and the relative expression levels were calculated using the $2^{-\Delta \Delta \mathrm{Ct}}$ method. GAPDH served as an

199 internal standard control. The primer and internal reference fragment sequences (5' -3') are as

200 Table 1.

201 8. Statistical analysis

202 All experiments were repeated three times. SPSS Statistics 20.0 software and GraphPad 203 Prism 7 were used to perform the statistical analyses. We used one-way ANOVA and the LSD-t 204 test to compare data between groups. Data are expressed as the mean \pm standard deviation, and 205 the differences with a $\mathrm{FC} \geq 2.0$ and a $p<0.05$ were considered statistically significant.

\section{Results}

1. Preparation of titanium

The TEM observation shows that the SMAT method can make the surface of gradient nanometal pure titanium nanocrystallization, and the grain size is on the nanometer scale (Fig. 1).

HBMSCs were observed as adherent cells under an inverted phase contrast microscope.

213 One day after passage, the cells were partially attached (Fig. 2A); after 3 days, most of the cells

214 were adherent, and the cell fusion degree was over $80 \%$. The morphology of the adherent cells 
215 changed from round to long fusiform or star-shaped, and the arrangement was spiral (Fig. 2B).

216 3. Proliferation curve of the hBMSCs

217 (1) After observing the hBMSC subculture for 7 days, the results showed that the hBMSCs

218 showed obvious latency, logarithmic proliferative and plateau phases, and the growth curve

219 assumed an "S" shape. Days 1-3 were the incubation period, and days 3-5 were the logarithmic

220 growth phase; On the 3rd and 5th day of culture, the difference between the SMAT titanium

221 group and the annealed titanium group was statistically significant $(p<0.05$; Fig. 2C).

222 4. Detection of ALP activity

223 When hBMSCs were cultured for 3, 5, 7 and 14 days, the ALP activity level in each group 224 increased over time. At each time point, the level of ALP activity in the SMAT titanium group 225 was higher than that in the annealed titanium group. On days 3 and 5, the differences between 226 the SMAT titanium group and the annealed titanium group were statistically significant $(p<0.05$; 227 Fig. 3D). It is shown that SMAT titanium can promote the early osteogenic differentiation of 228 hBMSCs.

229 5. Alizarin red staining

230 HBMSCs were induced osteogenically for 7, 14 and 21 days, and the alizarin red staining 231 area of each group increased over time. At each time point, the alizarin red staining area of the 232 SMAT titanium group was larger than that of the annealed titanium group. On the 14th day, the 233 difference between the SMAT titanium group and the annealed titanium group was statistically 234 significant $(p<0.05$; Fig. 3ABC and Fig. 3E). It is shown that SMAT titanium can promote the 235 late osteogenic differentiation of hBMSCs.

236 6. Biological information prediction

237 (1) Screening results for the osteogenesis-related miRNAs

238 According to the literature, 20 osteogenesis-related mRNAs have been identified, including 
ALK, COLIA1, RUNX2, SP7, IBSP, COL10VA1, BMP2, BGLAP, SPP1, DLX5, CBFB, BMP7, AcvR1b, LIF, INHBA, Noda1, BMP4, FGF2, IGF1, Wnt. Twelve mRNAs were confirmed to be

241 involved in the TGF- $\beta$ / Smad, MAPK/ ERK, Wnt, and Notch signalling pathways, including

242 Wnt3, Wnt4, Wnt8B, RUNX2, IGF1, ACVR1B, INHBC, CBFB, LIF, BMP7, NOG, CHRD.

243 Among them, Wnt3, Wnt4 and Wnt8B genes are involved in the Wnt signalling pathway, the

244 RUNX2 gene is involved in the Notch signalling pathway, the CBFB gene is involved in the

245 Smad2/3 signalling pathway, the LIF gene is involved in the ERK signalling pathway, the IGF1

246 is involved in the MAPK/ ERK signalling pathway, the INHBC, ACVR1B, NOG, CHRD genes

247 are involved in the TGF- $\beta$ signalling pathway, and the BMP7 gene is involved in the TGF- $\beta$ and

248 ERK signalling pathways (Fig. 4C-L).

249 Eight miRNAs were predicted, including hsa-miR-18b-5p, hsa-let-7b-5p, hsa-miR-1224-5p, 250 hsa-miR-129-5p, hsa-miR-145-5p, hsa-miR-24-3p, hsa-miR-5195-3p, hsa-miR-6088 (Table 2).

251 (2) Screening results for the osteogenesis-related circRNAs

252 Fifty-one circRNAs were screened by miwalk3.0 software (Table 3, Fig. 4AB).

253 (3) CeRNA network analysis of the osteogenesis-related circRNAs

254 Further analysis of the predicted 51 circRNAs revealed that 30 circRNAs and 8 miRNAs 255 constituted 6 ceRNA networks. Circ-GNB5/ circ-HERC1/ circ-KMT2A/ circ-LTBP2 interact 256 with hsa-miR-24-3p and Wnt3 gene in the Wnt signaling pathway; circ-TBC1D2B/ circ-TBCD/ 257 circ-TRIOBP/ circ-VPS13C interact with hsa-miR-6088 and CBFB gene in the Smad2/3 258 signaling pathway; circ-ABCA3 interacts with hsa-miR-18b-5p and CF2L2; circ-MDN1/ circ259 MYH9/ circ-PLPPR4/ circ-RAB31/ circ-SMARCH2/ circ-SPEG/ circ-SYNM/ circ-TANGO6 260 interact with hsa-miR-5195-3p and Wnt4 gene in the Wnt signaling pathway, the IGF1 gene in 261 the MAPK/ERK signaling pathway; hsa-miR-1224-5p combines with NOG gene in the TGF- $\beta$ 262 signaling pathway; circ-ADAMTS13/ circ-ARHGAP32/ circ-BRPF3/ circ-CARD8/ circ263 CCDC88C/ circ-COL4A2/ circ-DGKD/ circ-DIP2C/ circ-DYNC1H1/ circ-FAT3/ circ-LTBP2 
264 interact with hsa-let-7b-5p and CHRD gene in the TGF- $\beta$ signaling pathway (Fig 4M).

265 7. QRT-PCR assay

266 To verify the accuracy of the bioinformatics prediction, we screened circ-LTBP2 expression

267 by using QRT-PCR detection. The results of QRT-PCR showed that the expression levels of hsa268 circ-0032599, hsa-circ-0032600 and hsa-circ-0032601 were upregulated in the experimental 269 group, and the differential expression of hsa-circ-0032600 was the most upregulated and 270 statistically significant on the 14 th day $(\mathrm{FC}=4.25 \pm 1.60, p<0.05$; Fig. 5).

\section{Discussion}

273 SMAT technology uses high-energy balls to repeatedly impact the sample surface.

274 Typically, a gradient nanostructured surface layer, in which the grain size is in the nanometer 275 scale at the top surface and gradually increases into the micrometer scale in the interior, will be 276 achieved on metals by SMAT (T.H. Fang et al., 2011; Lu K, Lu J, 2004). In this study, the TEM 277 observation shows that the SMAT method can make the surface of gradient nano-metal pure 278 titanium nanocrystallization, and the grain size is on the nanometer scale. Since there is no 279 interface separating the surface layer and the substrate, the problem of poor interface bonding 280 between the nanostructured surface layer and the substrate, usually caused by traditional coating 281 techniques, is easily solved (Nana L, Ning W, 2018; Du H et al., 2019). In addition, SMAT 282 materials have the advantages of high strength, high hardness, high diffusion rate and high 283 chemical reactivity, and their wear resistance and fatigue resistance are also significantly 284 improved (Nowak W J, Serafin D, Wierzba B, 2019; Benafia S et al., 2018; Yao Q et al., 2017; 285 T.H. Fang et al., 2011; Z.B. Wang, K. Lu, 2017; W.P. Tong et al., 2003; Z.B. Wang et al., 2003; 286 Y.B. Lei et al., 2019; Lu K, Lu J, 2004). In this study, the influence of surface morphologies on 287 the adhesion, proliferation and differentiation of hBMSCs might be safely excluded, while the 288 samples in the experimental and controlled groups show a similar surface morphology (with 
289 surface roughness $R_{a}$ of $1.73 \pm 0.11$ and $1.82 \pm 0.06 \mu \mathrm{m}$, respectively). Therefore, it should be the

290 contribution from the surface nanostructure that the hBMSC osteogenic differentiation is

291 promoted on the SMAT titanium.

292 In this study, hBMSCs can differentiate into osteoblasts. A lot of literature have confirmed

293 that hBMSCs are important members of a family of stem cells and are derived from early

294 developing mesoderm and ectoderm. They are easy to extract and purify, and they still show

295 stem cell characteristics after repeated subculturing. They have strong proliferation and

296 differentiation ability and can be multi-directionally differentiated into osteoblasts, chondrocytes,

297 haematopoietic cells, muscle cells and other types of cells. They can be used to repair tissue and

298 organ damage (Saito A et al., 2018; Mohammadali F, Abroun S, Atashi A, 2018). They have a

299 haematopoietic support function, since they not only provide mechanical support for

300 haematopoietic stem cells in the bone marrow but also secrete a variety of growth factors, such

301 as interleukin (IL)-6, IL-11, leukaemia inhibitory actor (LIF), macrophage colony-stimulating

302 factor (M-CSF) and human stem cell growth factor (SCF), to support haematopoiesis (Amini A

303 et al., 2018; Mohammadali F, Abroun S, Atashi A, 2018). HBMSCs also regulate immune

304 function and do not show immunological characteristics consistent with rejection (Liu C et al.,

305 2018; Gabr M M et al., 2017; Lee H J et al., 2017).

306 To date, several osteogenesis-related signalling pathways have been documented, including

307 the TGF- $\beta$, Smad, MAPK, Wnt/ $\beta$-catenin, Notch, Hedgehog, fibroblast growth factor (FGF),

308 and orthopantomography (OPG)/ receptor activator for nuclear factor- $\kappa \mathrm{B}$ ligand (RANKL)

309 signalling pathways (Zhu W Q et al., 2018; Urbanek K et al., 2017; Jin L et al., 2017). TGF- $\beta$

310 can increase intracellular ALP activity and the synthesis and secretion of osteocalcin, collagen,

311 and osteonectin (Yang S et al., 2018). The TGF- $\beta$ pathway consists of extracellular ligands,

312 transmembrane receptors, and intracellular regulatory factors. The receptor TGF- $\beta$ activates

313 Smads and enters the nucleus, thereby regulating its target genes (Hui L et al., 2018); the MAPK

314 signalling pathway involves MAPK/ ERK/ big MAP kinase 1 (BMK1)/ stress activated protein

315 (SAPK)/ c-Jun N-terminal kinase (JNK)/ P38 in five main ways (Lou R et al., 2019; Jiang L et al.

Peer] reviewing PDF | (2019:12:44470:1:2:NEW 6 Apr 2020) 
316 2018). The MAPK signalling pathway also plays an important role in the proliferation,

317 differentiation and apoptosis of osteoblasts ( $\mathrm{Li} \mathrm{Z} \mathrm{H}$ et al., 2018). Wnt signalling plays an

318 important role in the proliferation and differentiation of mesenchymal stem cells,

319 osteoblastogenesis, bone formation, bone remodelling, and other processes (Wang X et al., 2019;

320 Shuai Y et al., 2019). The Wnt signalling pathway can also be involved in cell-to-cell signalling

321 through paracrine or autocrine signalling and participates in a variety of cellular processes, such

322 as cell proliferation, differentiation, polarization, and migration (Hang K et al., 2019; Jun-Li Liu

323 et al., 2017). The Notch signalling pathway involves the Notch receptor, DSL protein (ligand),

324 DNA binding protein and Notch regulatory molecule. It can play a role in multiple cell

325 morphogenetic processes, such as cell formation, differentiation, and apoptosis (Nandagopal $\mathrm{N}$ et

326 al., 2018; Sun Z et al., 2018; Carra S et al.,2017). In this study, we choose twelve osteogenesis-

327 related mRNAs associated with the TGF- $\beta$ / Smad, MAPK/ ERK, Wnt, and Notch signalling

328 pathways to predict osteogenesis-related miRNAs and circRNAs.

329 This study proves that there are differences in the expressions of osteogenesis-related

330 circRNAs in the process of SMAT titanium promoting the osteogenic differentiation of hBMSCs.

331 Currently, a large number of studies have shown that circRNA can regulate stem cell osteogenic

332 differentiation. Gu et al. cultured PDLSCs in osteogenic induction medium and normal medium

333 and found differences in the expression of a total of 1456 circRNAs by RNA sequencing. The

334 researchers used GO and KEGG analyses to predict that circRNA-BANP and circRNA-ITCH

335 can regulate the osteogenic differentiation of PDLSCs through the MAPK pathway (Gu X et al.,

336 2017). Li et al. (Xiaobei L et al., 2018) used qRT-PCR to detect the expression level of CDR1as

337 during the osteogenic differentiation of PDLSCs and constructed CDR1as overexpression and

338 silencing models to detect osteogenic differentiation. Animal experiments were performed to

339 analyse the formation of new bone by microcomputed tomography and various staining methods.

340 The results showed that CDR1as was upregulated and that silencing CDR1as could inhibit the

341 osteogenic differentiation of cells. In vivo experiments have shown that silencing CDR1as can

342 inhibit bone formation. 
343 Alkaline phosphatase is a sugar protease released by cytoplasmic special particles. It

344 hydrolyzes phosphate esters during osteogenesis, provides phosphoric acid for hydroxyapatite

345 deposition, and hydrolyzes pyrophosphate to relieve its inhibitory effect on bone salt formation.

346 Its activity level is positively correlated with the degree of osteogenic differentiation of cells. It is

347 an early marker of osteogenic differentiation of hBMSCs (Samuel S et al., 2016; Li R et al.,

348 2019). The sample was reacted in a carbonate buffer $(\mathrm{pH}=9.8)$ containing $\mathrm{p}$-nitrobenzene

349 phosphate. Under the action of alkaline phosphatase in the sample, p-nitrobenzene phosphate

350 was decomposed into p-nitrophenol and phosphoric acid. The resulting p-nitrophenol is basic

351 and yellow. Based on measuring the absorbance at $405 \mathrm{~nm}$, the alkaline phosphatase activity in

352 the sample was calculated. The hBMSCs on each group of materials gradually increased ALP

353 activity after osteogenesis induction, and the ALP activity of the SMAT group was higher than

354 that of the annealed group at each time point. On the 3rd and 5th days, the difference between the

355 SMAT group and the annealed group was statistically significant $(p<0.05)$. It is shown that

356 nanostructure can promote the early osteogenic differentiation of hBMSCs. Alizarin red is an

357 anionic dye that forms a red complex with metal ions. It can recognize and chelate calcium ions

358 to form orange-red calcium nodules. It is a sign of late osteogenic differentiation (Li R et al.,

359 2019). The area of alizarin red staining of cells on both groups of materials gradually increased

360 with the increase of culture time. At each time point of detection, the alizarin red staining area of

361 the SMAT group was larger than that of the annealed group. This shows that SMAT titanium has

362 better ability to promote the osteogenic differentiation of hBMSCs in late stage than annealed

363 titanium. On the 14th day, the difference between the SMAT group and the annealed group was

364 statistically significant $(p<0.05)$. It is shown that nanostructures can promote the late osteogenic

365 differentiation of hBMSCs.

366 In the predicted ceRNA networks, one combination of circ-GNB5, circ-HERC1, circ-

367 KMT2A, circ-LTBP2, hsa-miR-24-3p and the Wnt3 gene, another comprised of circ-LTBP2,

368 circ-MCF2L2, circ-MDN1, circ-MYH9, circ-PLPPR4, circ-RAB31, circ-SMARCH2, circ-SPEG,

369 circ-SYNM, circ-TANGO6, hsa-miR-5195-3p and the Wnt4, IGF1 genes, the third consist of 
370

371

372 three networks communicated with each other through the core hub circ-LTBP2. Based on the

373 previous prediction, it's target mRNAs including wnt3, wnt4, CBFB, IGF1 and CHRD are

374 recognized osteogenic marker genes (Kureel J et al., 2017; Qin X et al., 2015; Lindsey R C et al., 375 2018). What's more, circ-LTBP2 has not been reported yet. So we selected circ-LTBP2 for 376 verification.

377 In the comparison of the two groups of materials, QRT-PCR results showed that the 378 differential expression of hsa-circ-0032600 on the 14th day was significant (p-values), which is 379 consistent with the results of alizarin red staining. The combination mRNAs of hsa-circ-0032600 380 including wnt3, wnt4, CBFB, IGF1, CHRD involved in Wnt, Smad2/3, MAPK / ERK, TGF- $\beta$ 381 osteogenic pathways, which can regulate osteogenic differentiation in the middle and late stages 382 (Mobini S et al., 2017; Li R et al., 2019; Mao X Y et al., 2012). So hsa-circ-0032600 can 383 regulate the osteogenic differentiation of hBMSCs $\mathrm{n}$ the middle and late stages. However, vivo 384 experiments are still needed to confirm that hsa-circ-0032600 in promoting osteogenic 385 differentiation. And whether it can act as ceRNA, that is, the molecular sponge of miRNA, so as to reduce the effect of miRNA on gene expression still needs to be further investigated.

\section{Conclusions}

In this paper, we predict that the role of circ-LTBP2, which is shared by hsa-miR-24-3p, hsa-let-7b-5p and hsa-miR-5195-3p in osteogenic differentiation, still needs further experimental investigation. By using the prediction of the circRNAs that interact with 12 mRNAs and 8 miRNAs related to osteogenesis, it is necessary to further explore whether the relevant circRNAmiRNA-mRNA interactions function as sponges during osteogenic differentiation. 
395 to the KEGG analysis of circRNA parent genes, we identified 30 circRNAs and 8 miRNAs that

396 form 6 ceRNA networks. Moreover, the circRNAs located in key positions in the networks were

397 identified as being involved in the potential ceRNA mechanism of the circRNA-miRNA-mRNA

398 network, and their expression differences were verified by QRT-PCR analysis. To the best of our

399 knowledge, the role of hsa-circ-0032600 that was verified in this study has not yet been reported.

400 Therefore, this study demonstrates that SMAT titanium material can promote hBMSC osteogenic

401 differentiation by affecting circRNAs, and this material can be used clinically.

402

403 ACKNOWLEDGEMENTS

404 We thank Mingshan Li for guiding us about the bioinformatics.

405 ADDITIONAL INFORMATION AND DECLARATIONS

\section{Funding}

407 This study was supported by National Natural Science Foundation of China (No. 81970980),

408 Liaoning Provincial Key Research Plan Guidance Project (No. 2018225078), Liaoning

409 Provincial Natural Science Foundation Guidance Project (NO. 2019-ZD-0749) and Shenyang

410 Major Scientific and Technological Innovation Research and Development Plan (NO. 19-112-4-

411 027), Shenyang National Laboratory for Materials Science (No. 2015RP04). The funders had no

412 role in study design, data collection and analysis, decision to publish, or preparation of the

413 manuscript.

\section{Grant Disclosures}

415 The authors declare that they have no grant disclosures.

\section{Competing Interests}

417 The authors declare that they have no competing interests. 


\section{Author Contributions}

419 Wei Wang conceived and designed the experiments, and helped improve this paper.

420 Shanshan Zhu and Yuhe Zhu collected the samples, performed the experiments, analyzed the

421 data, prepared the figures and tables, and wrote this paper.

422 Jie Liu contributed the equipments and analysis tools, and revised this paper critically for 423 important content.

424 Zhenbo Wang participated in designing experimental routes and writing the manuscript 425 (preparation and microstructure of Ti samples).

426 Chen Liang helped prepare the SMAT and annealed Ti samples.

427 Nanjue Cao, Mingyan and Feigao helped improve the figures.

429 References

430 1. Camargo WA, Takemoto S, Hoekstra JW, Leeuwenburgh SC.G, Jansen JA, Beucken

431 JJ.J.P.vd, Alghamdic HS. 2017. Effect of surface alkali-based treatment of titanium implants 432 on ability to promote in vitro mineralization and in vivo bone formation. ACTA

433 BIOMATERIALIA 57:511 DOI:10.1016/j.actbio.2017.05.016.

434 2. Song P, Hu C, Pei X, Sun J, Sun H, Wu L, Jiang Q, Fan H, Yang B, Zhou C, Fan Y,

435 Zhang X. 2019. Dual modulation on crystallinity and macro/micro structures of 3D printed

436 porous titanium implants to enhance the stability and osseointegration. Journal of Materials 437 Chemistry B 7 DOI:10.1039/C9TB00093C.

438 3. Duan M, Wu X, Yuan L, Zhang Z, Zhang Y, Zhou Y. 2019. Fabrication and In vitro

439 Bioactivity of Robust Hydroxyapatite Coating on Porous Titanium Implant. Chemical

440 Research in Chinese Universities 35: 686-692 DOI:10.1007/s40242-019-9101-x. 
441 4. Zhou W, Huang O, Gan Y, Li Q, Zhou T, Xi W. 2019. Effect of titanium implants

442 with coatings of different pore sizes on adhesion and osteogenic differentiation of BMSCs.

443 Artificial Cells, Nanomedicine, and Biotechnology 47:290-299.

444 5. Sven H, Christoph A, Tarek E, Deml M, Milavec H, Bigdon SF, Benneker LM. 2018.

445 First Results of a New Vacuum Plasma Sprayed (VPS) Titanium-Coated Carbon/PEEK

446 Composite Cage for Lumbar Interbody Fusion. Journal of Functional Biomaterials 9:23

447 DOI:10.3390/jfb9010023.

448 6. Ito T, Ohtsu N, Tomozawa M, Takita H, Iizuka T, Yokoyama A. 2018. Promotion of

449 bone regeneration on titanium implants through a chemical treatment process using calcium

450 phosphate slurry: Microscopic analysis, cellular response, and animal experiment. Journal of

451 Biomedical Materials Research Part B Applied Biomaterials 106: 2716-2724

452 DOI:10.1002/jbm.b.34089.

453 7. Minko D, Belyavin K, Sheleg V. 2017. Biomechanical properties of composite

454 compact-porous titanium produced by electric discharge dintering. IOP Conference Series

455 Materials Science and Engineering DOI:10.1088/1757-899X/218/1/012008.

456 8. Lai M, Cai K, Hu Y, Yang X, Liu Q. 2012. Regulation of the behaviors of mesenchymal

457 stem cells by surface nanostructured titanium. Colloids Surf. B 97:211-220

458 DOI:10.1016/j.colsurfb.2012.04.029.

459 9. T.H. Fang, W.L. Li, N.R. Tao, K. Lu. 2011. Revealing extraordinary intrinsic tensile

460 plasticity in gradient nano-grained copper. Science 331:1587-1590

461 DOI:10.1126/science. 1200177.

462 10. Z.B. Wang, K. Lu. 2017. Diffusion and surface alloying of gradient nanostructured

463 metals. Beilstein J. Nanotechnol 8:547-560 DOI: 10.3762/bjnano.8.59.

464 11. W.P. Tong, N.R. Tao, Z.B. Wang, J. Lu, K. Lu. 2003. Nitriding iron at lower 
465 temperatures. Science 299:686-688 DOI: 10.1126/science.1080216.

466 12. Z.B. Wang, N.R. Tao, S. Li, W. Wang, G. Liu, J. Lu, K. Lu. 2003. Effect of surface

467 nanocrystallization on friction and wear properties in low carbon steel. Mater. Sci. Eng. A 468 352:144-149 DOI:10.1016/S0921-5093(02)00870-5.

469 13. Y.B. Lei, Z.B. Wang, J.L. Xu, K. Lu. 2019. Simultaneous enhancement of stress-

470 and strain-controlled fatigue properties in 316L stainless steel with gradient nanostructure. 471 Acta Mater 168:133-142.

472 14. Lu K, Lu J. 2004. Nanostructured surface layer on metallic materials induced by

473 surface mechanical attrition treatment. Mater. Sci. Eng. A 375-377:38-45

474 DOI:10.1016/j.msea.2003.10.261.

475 15. Son JW, Kim BC, Yoon JH, Lee J, Lim HJ. 2018. Mandibular Regeneration With

476 Autologous Human Bone Marrow Derived Mesenchymal Stem Cells to Treat Unicystic

477 Ameloblastoma. Journal of Craniofacial Surgery 30:1 DOI:10.1097/SCS.0000000000004917

478 16. Miranda H C, Herai R H, Carolina Hassibe Thomé, Gomes GG, Panepucci RA,

479 Orellana MD, Covas DT, Muotri AR, Greene LJ, Faça VM. 2012. A quantitative

480 proteomic and transcriptomic comparison of human mesenchymal stem cells from bone

481 marrow and umbilical cord vein. PROTEOMICS 12:2607-2617

482 DOI:10.1002/pmic.201200111.

483 17. Zhao R, Li F Q, Tian L L, Shang DS, Guo Y, Zhang JR, Liu M. 2019. Comprehensive 484 analysis of the whole coding and non-coding RNA transcriptome expression profiles and 485 construction of the circRNA-lncRNA co-regulated ceRNA network in laryngeal squamous 486 cell carcinoma. Functional \& Integrative Genomics 19:109-121 DOI:10.1007/s10142-018$487 \quad 0631-\mathrm{y}$.

488 18. Ji HN, Kim KM, Mcclusky WG, Abdelmohsen K, Gorospe M. 2018. Cytoplasmic 
489 functions of long noncoding RNAs. Wiley Interdisciplinary Reviews Rna 9:e1471

$490 \quad$ DOI:10.1002/wrna.1471.

491 19. Liu J, Liu T, Wang X, He A. 2017. Circles reshaping the RNA world: from waste

492 to treasure. Molecular Cancer 16:58 DOI:10.1186/s12943-017-0630-y.

493 20. Zhao Z, Wang K, Wu F, Wang W, Zhang K, Hu H, Liu Y, Jiang T. 2018. circRNA disease:

494 a manually curated database of experimentally supported circRNA-disease associations.

495 Cell Death \& Disease, 9:475.

496 21. Zhang M, Xin Y. 2018. Circular RNAs: a new frontier for cancer diagnosis and therapy.

$497 \quad$ Journal of Hematology \& Oncology 11:21.

498 22. Kulcheski F R, Christoff A P, Margis R. 2016. Circular RNAs are miRNA sponges and

499 can be used as a new class of biomarker. Journal of Biotechnology

$500 \quad$ 238:S0168165616315292 DOI:10.1016/j.jbiotec.2016.09.011.

501 23. Cortéslópez M, Gruner M R, Cooper D A, Gruner HN, Voda AI, Linden AMvd, Miura P.

502 2018. Global accumulation of circRNAs during aging in Caenorhabditis elegans. Bmc

503 Genomics 19:8 DOI:10.1186/s12864-017-4386-y.

504 24. Li S, Han L. 2019. Circular RNAs as promising biomarkers in cancer: detection, function, 505 and beyond. Genome Medicine 11:15 DOI:10.1186/s13073-019-0629-7.

506 25. Mengjun Z, Lingfei J, Yunfei Z. 2018. circRNA Expression Profiles in Human Bone 507 Marrow Stem Cells Undergoing Osteoblast Differentiation. Stem Cell Reviews and Reports $508 \quad$ 15:126-138 DOI:10.1007/s12015-018-9841-X.

509 26. Zheng Y, Li X, Huang Y, Jia L, Li W. 2017. The Circular RNA Landscape of Periodontal 510 Ligament Stem Cells During Osteogenesis. Journal of Periodontology 88:906-914 511 DOI:10.1902/jop.2017.170078.

512 27. Nana L, Ning W. 2018. The effect of duplex Surface mechanical attrition and nitriding 513 treatment on corrosion resistance of stainless steel 316L. Scientific Reports 8:8454 514 DOI:10.1038/s41598-018-26844-0. 
515 28. Du H, An Y, Zhang X, Wei Y, Hou L, Liu H, Zhang J, Wang N, Umar A, Guo Z. 2019.

516 Hydroxyapatite (HA) Modified Nanocoating Enhancement on AZ31 Mg Alloy by

517 Combined Surface Mechanical Attrition Treatment and Electrochemical Deposition

518 Approach. Journal of Nanoscience and Nanotechnology 19:810-818

$519 \quad$ DOI:10.1166/jnn.2019.15746.

520 29. Nowak W J, Serafin D, Wierzba B. 2019. Effect of surface mechanical treatment on the 521 oxidation behavior of FeAl-model alloy. Journal of Materials Science, 54:9185-9196.

522 30. Benafia S, Retraint D, Brou S Y. 2018. Influence of Surface Mechanical Attrition

523 Treatment on the oxidation behaviour of 316L stainless steel. Corrosion Science 136:188524200 .

525 31. Yao Q, Sun J, Zhang G, Tong W, Zhang H. 2017. Thermal Stability of Nanocrystalline 526 BCC-Ti Formed by Phase Transformation During Surface Mechanical Attrition Treatment. $527 \quad$ Nano 12:1750113 DOI:10.1142/S1793292017501132.

528 32. Saito A, Nagaishi K, Iba K, Yuka Mizue, Chikenji T, Otani M, Nakano M, Oyama K, 529 Yamashita T, Fujimiya M. 2018. Umbilical cord extracts improve osteoporotic 530 abnormalities of bone marrow-derived mesenchymal stem cells and promote their 531 therapeutic effects on ovariectomised rats. Scientific Reports 8:1161 DOI:

$532 \quad 10.1038 / \mathrm{s} 41598-018-19516-6$.

533 33. Mohammadali F, Abroun S, Atashi A. 2018. Mild hypoxia and human bone marrow 534 mesenchymal stem cells synergistically enhance expansion and homing capacity of human 535 cord blood CD34+ stem cells. Iranian Journal of Basic Medical Sciences 21:709-716 DOI:

$536 \quad 10.22038 / \mathrm{ijbms.2018.26820.6561.}$

537 34. Amini A, Pouriran R, Abdollahifar M A, Abbaszadeh HA, Ghoreishi SK, Chien S, Bayat 538 M. 2018. Stereological and molecular s tudies on the combined effects of

539 photobiomodulation and human bone marrow mesenchymal stem cell conditioned medium 540 on wound healing in diabetic rats. Journal of Photochemistry and Photobiology B: Biology $541 \quad 182: 42-51$. 
542

543

544

545

546

547

548

549

550

551

552

553

554

555

556

557

558

559

560

561

562

563

564

565

566

567

35. Mohammadali F, Abroun S, Atashi A. 2018. Mild hypoxia and human bone marrow mesenchymal stem cells synergistically enhance expansion and homing capacity of human cord blood CD34+ stem cells.Iranian Journal of Basic Medical Sciences 21:709-716 DOI:

\subsection{8/ijbms.2018.26820.6561.}

36. Liu C, Feng X, Wang B, Wang X, Wang C, Yu M, Cao G, Wang H. 2018. Bone marrow mesenchymal stem cells promote head and neck cancer progression through Periostin-mediated phosphoinositide 3-kinase/Akt/mammalian target of rapamycin. Cancer Science 109:688-698 DOI:10.1111/cas.13479.

37. Gabr MM, Zakaria MM, Refaie AF, Rahman EAA, Rahman EAA, Reda AM. Ali SS,

Khater SM, Ashamallah SA, Ismail AM, Ismail HEDA, Badri NE, Ghoneim MA. 2017. From Human Mesenchymal Stem Cells to Insulin-Producing Cells: Comparison between Bone Marrow- and Adipose Tissue-Derived Cells. Biomed Research International 2017:1-9 DOI:10.1155/2017/3854232.

38. Lee H J, Kim S N, Jeon M S, Yi TG, Song SU. 2017. ICOSL expression in human bone marrow-derived mesenchymal stem cells promotes induction of regulatory $\mathrm{T}$ cells. Scientific Reports 7:44486 DOI:10.1038/srep44486.

39. Zhu W Q, Ming P P, Jing Q, Shao SY, Yu YJ, Chen JX, Yang J, Xu LN, Zhang SM, Tang CB. 2018. Effect of titanium ions on the Hippo/YAP signaling pathway in regulating biological behaviors of MC3T3-E1 osteoblasts. Journal of Applied Toxicology 38:824-833 DOI:10.1002/jat.3590.

40. Urbanek K, Lesiak M, Krakowian D, Komarska HK, Likus W, Czekaj P,Kusz D, Sieroń AL. 2017. Notch signaling pathway and gene expression profiles during early in vitro differentiation of liver-derived mesenchymal stromal cells to osteoblasts. Laboratory Investigation 97:1225-1234 DOI:10.1038/labinvest.2017.60.

41. Jin L, Cao Y, Yu G, Wang J, Lin X, Ge L, Du J, Wang L, Diao S, Lian X, Wang S, Dong R, Shan Z. 2017. SFRP2 enhances the osteogenic differentiation of apical papilla 
568

569

570

571

572

573

574

575

576

577

578

579

580

581

582

583

584

585

586

587

588

589

590

591

592

593

594

stem cells by antagonizing the canonical WNT pathway. Cellular \& Molecular Biology Letters 22:14 DOI:10.1186/s11658-017-0044-2.

42. Yang S, Yu Y, Shuang Z, Wang J, Xiao Y, Liu C. 2018. The immunomodulatory role of sulfated chitosan in BMP-2-mediated bone regeneration. Biomaterials Science 6:2496-2507 DOI:10.1039/C8BM00701B.

43. Hui L, Zhang S, Nie B, Long T, Qu X, Yue B. 2018. The antimicrobial peptide KR-12 promotes the osteogenic differentiation of human bone marrow stem cells by stimulating BMP/SMAD signaling. Rsc Advances 8:15547-15557 DOI:10.1039/c8ra00750k.

44. Lou R, Dong S, Shi C, Shi C, Xu X, Ma R, Wu J, Feng J. 2019. Liver X receptor agonist T0901317 inhibits the migration and invasion of non-small-cell lung cancer cells in vivo and in vitro. Anticancer Drugs 30:495-500 DOI:10.1097/CAD.0000000000000758.

45. Jiang L, Wang Y, Liu G, Liu H, Zhu F, Ji H, Li B. 2018. C-Phycocyanin exerts anti-cancer effects via the MAPK signaling pathway in MDA-MB-231 cells. Cancer Cell International 18:12 DOI:10.1186/s12935-018-0511-5.

46. Li Z H, Li L, Kang L P, Wang Y. 2018. MicroRNA-92a promotes tumor growth and suppresses immune function through activation of MAPK/ERK signaling pathway by inhibiting PTEN in mice bearing U14 cervical cancer. Cancer Medicine 7: 3118-3131 DOI:10.1002/cam4.1329.

47. Wang X, Wang X, Liu Y, Dong Y, Wang Y, Kassab MA, Fan W, Yu X, Wu C. 2019. LGR5 regulates gastric adenocarcinoma cell proliferation and invasion via activating Wnt signaling pathway. Oncogenesis 8:57 DOI:10.1038/s41389-018-0071-5.

48. Shuai Y, Yang R, Mu R, Yu Y, Rong L, Jin L. 2019. MiR-199a-3p mediates the adipogenic differentiation of bone marrow-derived mesenchymal stem cells by regulating KDM6A/WNT signaling. Life Sciences 220:84-91 DOI:10.1016/j.1fs.2019.01.051.

49. Hang K, Ye C, Xu J, Chen E, Wang C, Zhang W, Ni L, Kuang Z, Ying K, Xue D, Pan Z. 2019. Apelin enhances the osteogenic differentiation of human bone marrow mesenchymal stem cells partly through Wnt/ $\beta$-catenin signaling pathway. Stem Cell Research \& Therapy 
596

597

598

599

600

601

602

603

604

605

606

607

608

609

610

611

612

613

614

615

616

617

618

619

620

621

50. Jun-Li Liu, Kaddour N, Chowdhury S, Li Q, Gao ZH. 2017. Role of CCN5 (WNT1 inducible signaling pathway protein 2) in pancreatic islets. Journal of Diabetes 9:462-474 DOI:10.1111/1753-0407.12507.

51. Nandagopal N, Santat LA, LeBon L, Sprinzak D, Bronner ME, Elowitz MB. 2018. Dynamic Ligand Discrimination in the Notch Signaling Pathway. Cell 172:S0092867418300023 DOI:10.1016/j.cell.2018.01.002.

52. Sun Z, Cheng Z, Feng L, Zhang W, Chen J, Pan Y, Ma L, Liu Q, Du Y, Yang J, Wang Q. 2018. Inhibition of breast cancer cell survival by Xanthohumol via modulation of the Notch signaling pathway in vivo and in vitro. Oncology Letters 15:908-916 DOI:10.3892/ol.2017.7434.

53. Carra S, Sangiorgio L, Pelucchi P, Cermenati S, Mezzelani A, Martino V, Palizban M, Albertini A, Götte M, KehlerJ, Deflorian G, Beltrame M, Giordano A, Reinbold R, Cote lli F, Bellipanni G, Zucchi I. 2017. Zebrafish Tmem230a cooperates with the Delta/Notch signaling pathway to modulate endothelial cell number in angiogenic vessels. Journal of Cellular Physiology 233:1455-1467 DOI:10.1002/jcp.26032.

54. Gu X, Li M, Jin Y, Liu D, Wei F. 2017. Identification and integrated analysis of differentially expressed lncRNAs and circRNAs reveal the potential ceRNA networks during PDLSC osteogenic differentiation. BMC Genetics 18:100 DOI:10.1186/s12863-0170569-4.

55. Xiaobei L, Yunfei Z, Yan Z, Huang Y, Zhang Y, Jia L, Li W. 2018. Circular RNA CDR1as regulates osteoblastic differentiation of periodontal ligament stem cells via the miR-7/GDF5/SMAD and p38 MAPK signaling pathway. Stem Cell Research \& Therapy 9:232 DOI:10.1186/s13287-018-0976-0.

56. Samuel S, Ahmad RE, Ramasamy TS, Karunanithi P, Naveen SV, Murali MR, Abbas AA, Kamarul T. 2016. Platelet-rich concentrate in serum free medium enhances osteogenic differentiation of bone marrow-derived human mesenchymal stromal cells. PeerJ 4:e2347 
622 DOI:10.7717/peerj.2347.

623 57. Li R, Sun J, Yang F, Sun Y, Wu X, Zhou Q, Yu Y, Bi W. 2019. Effect of GARP on

624 osteogenic differentiation of bone marrow mesenchymal stem cells via the regulation of 625 TGFß1 in vitro. PeerJ 7:e6993 DOI:10.7717/peerj.6993.

626 58. Kureel J, John A A, Prakash R, Singh D. 2017. MiR 376c inhibits osteoblastogenesis by

627 targeting Wnt3 and ARF-GEF-1 facilitated augmentation of beta-catenin transactivation.

628 Journal of Cellular Biochemistry 119: 3293-3303 DOI:10.1002/jcb.26490.

629 59. Qin X, Jiang Q, Matsuo Y, Kawane T, Komori H, Moriishi T, Taniuchi I, Ito K, Kawai

630 Y, Rokutanda S, Izumi S, Komori T. 2015. Cbfb regulates bone development by

631 stabilizing runx family proteins. Journal of Bone and Mineral Research 30: 706-714

632 DOI:10.1002/jbmr.2379.

633 60. Lindsey R C , Rundle C H , Mohan S. 2018. Role of IGF-I and Efn-Eph signaling

634 in skeletal metabolism. Journal of Molecular Endocrinology 61:JME-17-0284 DOI:

635 10.1530/JME-17-0284.

636 61. Mobini S, Leppik L, Thottakkattumana Parameswaran V, Barker JH. 2017. In

637 vitro effect of direct current electrical stimulation on rat mesenchymal stem

638 cells. PeerJ 5:e2821 DOI:10.7717/peerj.2821.

639 62. Li R, Sun J, Yang F, Sun Y, Wu X, Zhou Q, Yu Y, Bi W. 2019. Effect of GARP on

640 osteogenic differentiation of bone marrow mesenchymal stem cells via the regulation of

641 TGFß1 in vitro. PeerJ 7:e6993 DOI:10.7717/peerj.6993

642 63. Mao X Y, Bian Q, Shen Z Y. 2012. Analysis of the osteogenetic effects exerted on 643 mesenchymal stem cell strain C3H10T1/2 by icariin via MAPK signaling pathway in vitro. 644 Journal of Chinese Integrative Medicine 10:1272-1278 DOI:10.4236/ojemd.2015.52002. 
646

647

Peer] reviewing PDF | (2019:12:44470:1:2:NEW 6 Apr 2020) 


\section{Table 1 (on next page)}

Primer and internal reference gene sequence table. 
1 Table 1:

2 Primer and internal reference gene sequence table.

\begin{tabular}{|c|c|c|}
\hline $\begin{array}{c}\text { Name of circRNA/ internal } \\
\text { reference }\end{array}$ & Primer sequence (5',3') & Product length (bp) \\
\hline hsa-circ-0032599 & CTGGAACCTGCGTGAACCT & 226 \\
\hline TGATCCGCTGGGCCAAAG & 221 \\
\hline hsa-circ-0032600 & CTGGAACCTGCGTGAACCT & \\
\hline TCTTGGCAGTGAGTGAGGTT & 229 \\
\hline GAPDH & CTGGAACCTGCGTGAACCT & \\
\hline & TGGTGGATCTGCACTGAGG & \\
\hline
\end{tabular}

3 


\section{Table 2 (on next page)}

Predicted miRNAs and their gene symbols, signaling pathways. 
Table 2 Predicted miRNAs and their gene symbols, signaling pathways.

\begin{tabular}{|c|c|c|}
\hline miRNA & gene symbol & signaling pathway \\
\hline hsa-miR-18b-5p & $\mathrm{ABCA} 3$ & \\
\hline hsa-let-7b-5p & HERC1 & \\
\hline hsa-miR-1224-5p & MCF2L2、PLPPR4、SPEG & \\
\hline \multirow{4}{*}{ hsa-miR-129-5p } & FAT3、LTBP2、MYH9、 & \\
\hline & SMARCA2、TBC1D2B、 & \\
\hline & VPS13C、DYNC1H1 & \\
\hline & DGKD & Phospholipase D 、 Gastrin-CREB via PKC and MAPK \\
\hline \multirow{2}{*}{ hsa-miR-145-5p } & BRPF3、TANGO6 & \\
\hline & COL4A2 & AGE-RAGE、ERK 、 FAK1 、GnRH 、ILK \\
\hline hsa-miR-24-3p & $\begin{array}{c}\text { ADAMTS13、DIP2C、 } \\
\text { SYNM }\end{array}$ & \\
\hline \multirow{3}{*}{ hsa-miR-5195-3p } & $\begin{array}{l}\text { ARHGAP32、CCDC88C、 } \\
\text { FLNA、LTBP2、MDN1、 }\end{array}$ & \\
\hline & RAB31、TBCD、TRIOBP & \\
\hline & GNB5 & $\begin{array}{l}\text { Corticotropin-releasing hormone 、 G Protein 、 } \\
\text { Apelin 、Chemokine 、 Chemotaxis CXCR4 }\end{array}$ \\
\hline \multirow{2}{*}{ hsa-miR-6088 } & CARD8 & NOD-like receptor \\
\hline & KMT2A & \\
\hline hsa-miR-18b-5p & $\mathrm{ABCA} 3$ & \\
\hline hsa-let-7b-5p & HERC1 & \\
\hline \multirow[t]{2}{*}{ hsa-miR-1224-5p } & MCF2L2、PLPPR4、SPEG & \\
\hline & FAT3、LTBP2、MYH9、 & \\
\hline hsa-miR-129-5p & SMARCA2、TBC1D2B、 & \\
\hline
\end{tabular}


DGKD BRPF3、TANGO6

hsa-miR-145-5p

3

hsa-miR-24-3p

hsa-miR-6088
COL4A2 ADAMTS13、DIP2C、 SYNM ARHGAP32、CCDC88C、 FLNA、LTBP2、MDN1、 RAB31、TBCD、TRIOBP

GNB5

CARD8

KMT2A
Corticotropin-releasing hormone 、 G Protein 、

Apelin 、Chemokine 、 Chemotaxis CXCR4

NOD-like receptor 


\section{Table 3(on next page)}

Predicted circRNAs and their chrs, gene symbols, miRNAs, signaling pathways. 
1 Table 3 Predicted circRNAs and their chrs, gene symbols, miRNAs, signaling pathways.

2

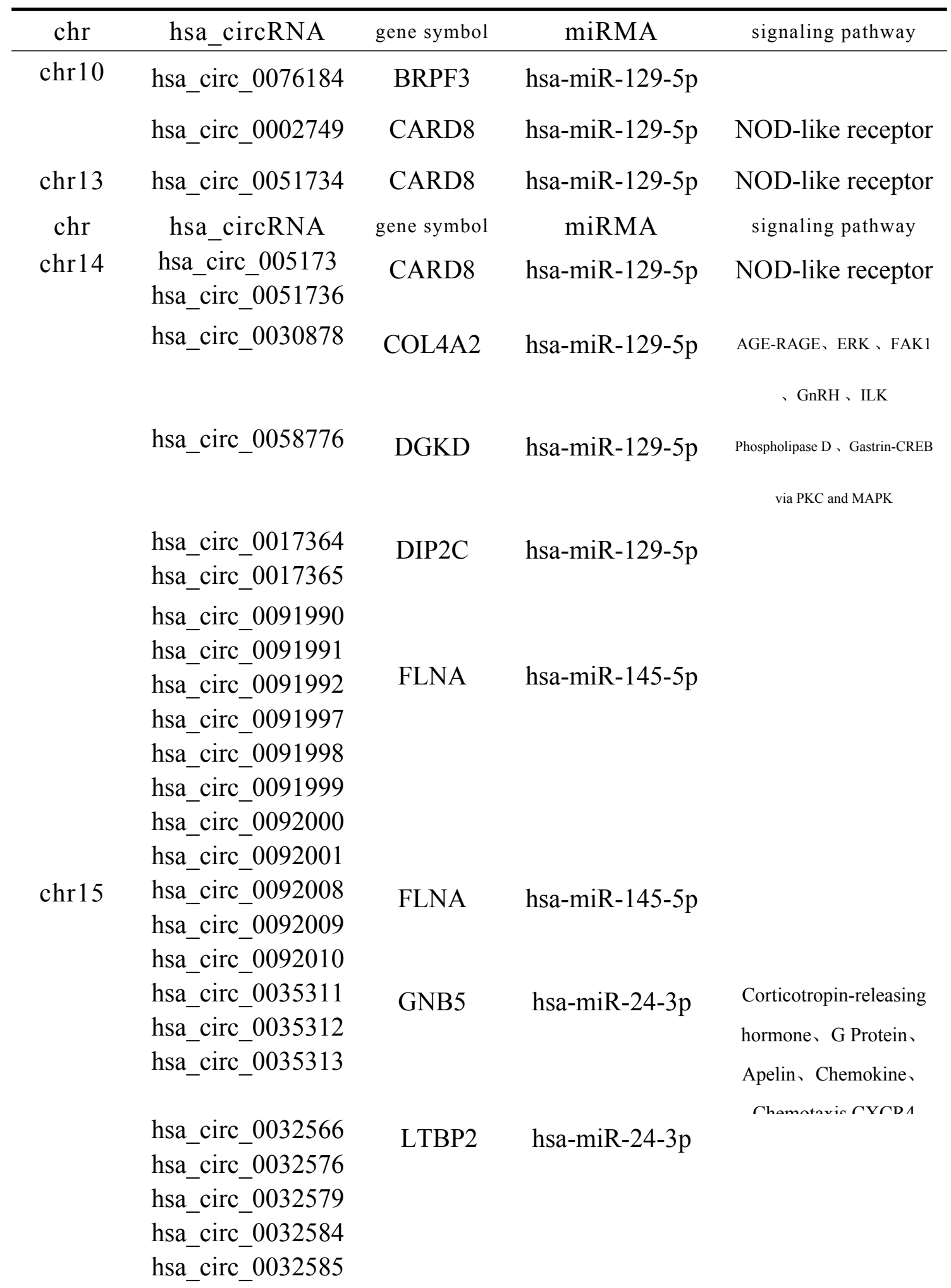


hsa_circ_0032594

$\operatorname{chr} 17$

hsa_circ_0032599

hsa_circ_0032600

hsa_circ_0032601

hsa_circ_0032602

hsa_circ_0032604

hsa_circ_0032605

hsa_circ_0032607

hsa_circ_0032608

\begin{tabular}{|c|c|c|c|}
\hline & hsa_circRNA & gene symbol & miRMA \\
\hline $\operatorname{chr} 17$ & $\begin{array}{l}\text { hsa_circ_0077356 } \\
\text { hsa_circ_0077360 } \\
\text { hsa_circ_0077371 }\end{array}$ & MDN1 & hsa-miR-5195-3p \\
\hline $\operatorname{chr} 18$ & hsa_circ_0077375 & MDN1 & hsa-miR-5195-3p \\
\hline & hsa_circ_0063112 & MYH9 & hsa-miR-5195-3p \\
\hline $\operatorname{chr} 19$ & $\begin{array}{l}\text { hsa_circ_0063114 } \\
\text { hsa_circ_0063115 } \\
\text { hsa_circ_0063117 } \\
\text { hsa_circ_0046890 }\end{array}$ & MYH9 & hsa-miR-5195-3p \\
\hline $\operatorname{chr} 2$ & hsa_circ_0046891 & RAB31 & \\
\hline $\operatorname{chr} 22$ & hsa_circ_0037043 & SYNM & hsa-miR-5195-3p \\
\hline & $\begin{array}{l}\text { hsa_circ_0036435 } \\
\text { hsa_circ_0036436 } \\
\text { hsa_circ_0036437 }\end{array}$ & TBC1D2B & hsa-miR-6088 \\
\hline chr6 & $\begin{array}{l}\text { hsa_circ_0036438 } \\
\text { hsa_circ_0036439 } \\
\text { hsa_circ_0036440 } \\
\text { hsa_circ_0036441 }\end{array}$ & TBC1D2B & hsa-miR-6088 \\
\hline & hsa_circ_0046529 & TBCD & hsa-miR-6088 \\
\hline chrx & $\begin{array}{l}\text { hsa_circ_0046568 } \\
\text { hsa_circ_0046569 } \\
\text { hsa_circ_0046577 } \\
\text { hsa_circ_0046588 } \\
\text { hsa_circ_0046589 } \\
\text { hsa_circ_0046590 } \\
\text { hsa_circ_0046591 }\end{array}$ & TBCD & hsa-miR-6088 \\
\hline
\end{tabular}

\section{LTBP2 hsa-miR-24-3p}

signaling pathway 
hsa_circ_0046594

hsa_circ_0046595

hsa_circ_0046596

hsa_circ_0035596 VPS13C hsa-miR-6088

3 


\section{Figure 1}

The TEM observation of the gradient nano-metal pure titanium.

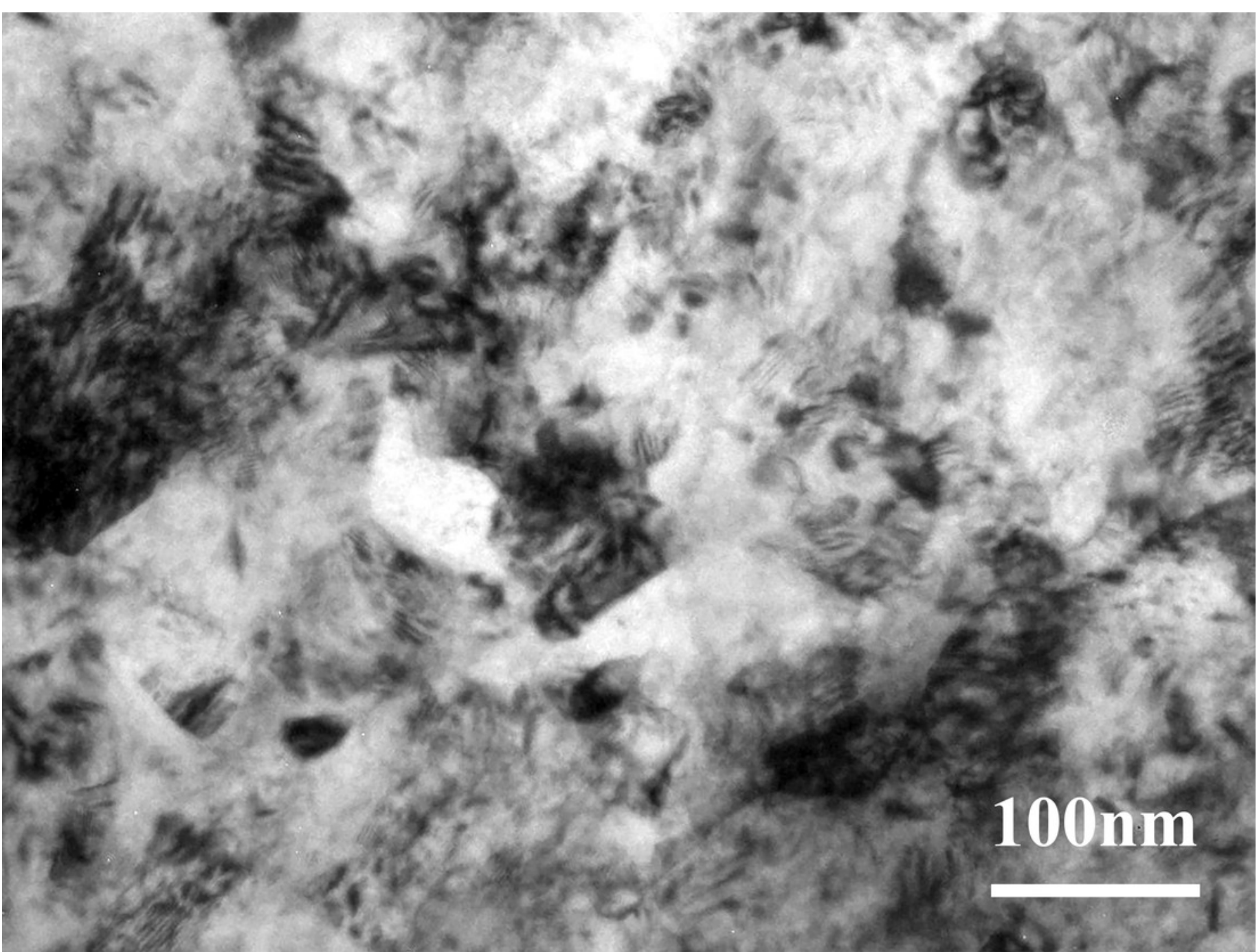




\section{Figure 2}

Culture and proliferation curve of the hBMSCs.

(A) HBMSCs cultured for 1 day (x100). (B) HBMSCs cultured for 3 days (x100). (C) CCK8 detection at 1, 3, 5, and 7 days of hBMSCs culture. *: The SMAT group compared with the annealed group. (mean $\pm \mathrm{SD}, \mathrm{n}=3, *$ indicates $p<0.05$ ).
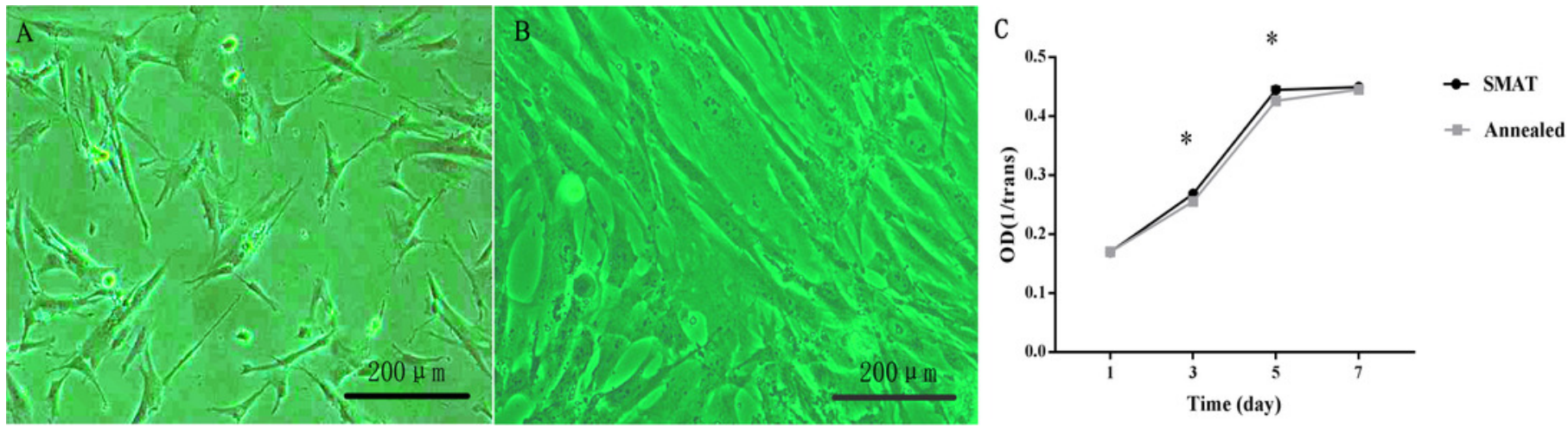
Figure 3

Detection of ALP activity and alizarin red staining of hBMSCs.

(A) Alizarin red staining after osteogenic induction of hBMSCs for 7 days, (B) 14 days and (C) 21 days. (D) Detection of ALP activity at 3, 5, 7 and 14 days after osteogenic induction of hBMSCs. *: The SMAT group compared with the annealed group. (mean $\pm \mathrm{SD}, \mathrm{n}=3$, * indicates $p<0.05)$. (E) The area of alizarin red staining at 7, 14, 21 days after osteogenic induction of hBMSCs. *: The SMAT group compared with the annealed group. (mean \pm SD, $n$ $=3, *$ indicates $p<0.05)$.

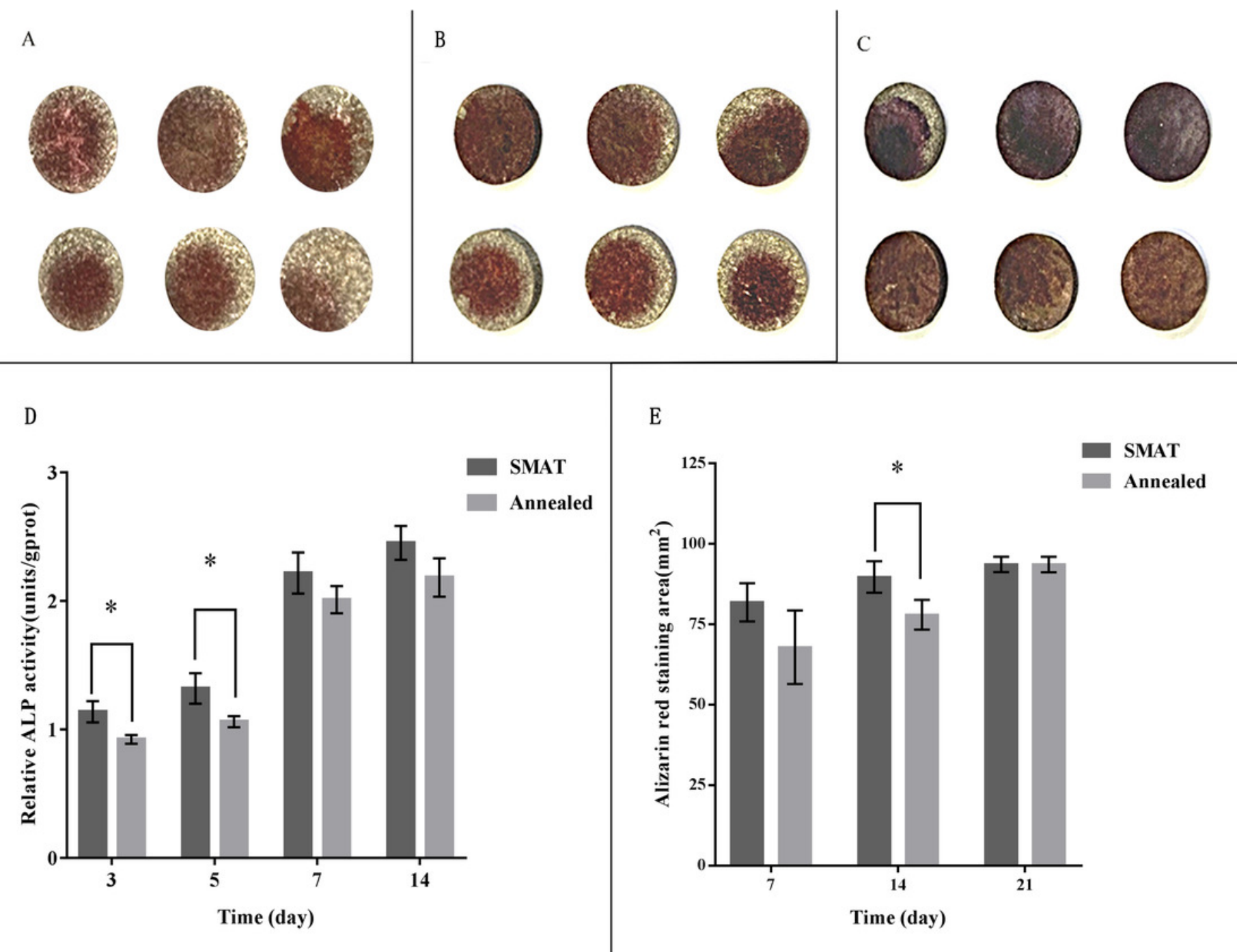




\section{Figure 4}

Biological information prediction.

(A) Numbers of circRNAs corresponding to different chrs. (B) Numbers of circRNAs on different signaling pathways. (C-L) Genes in the signaling pathways. (M) Network analysis of osteogenesis-related miRNAs and their interacting circRNAs and target genes. 
A

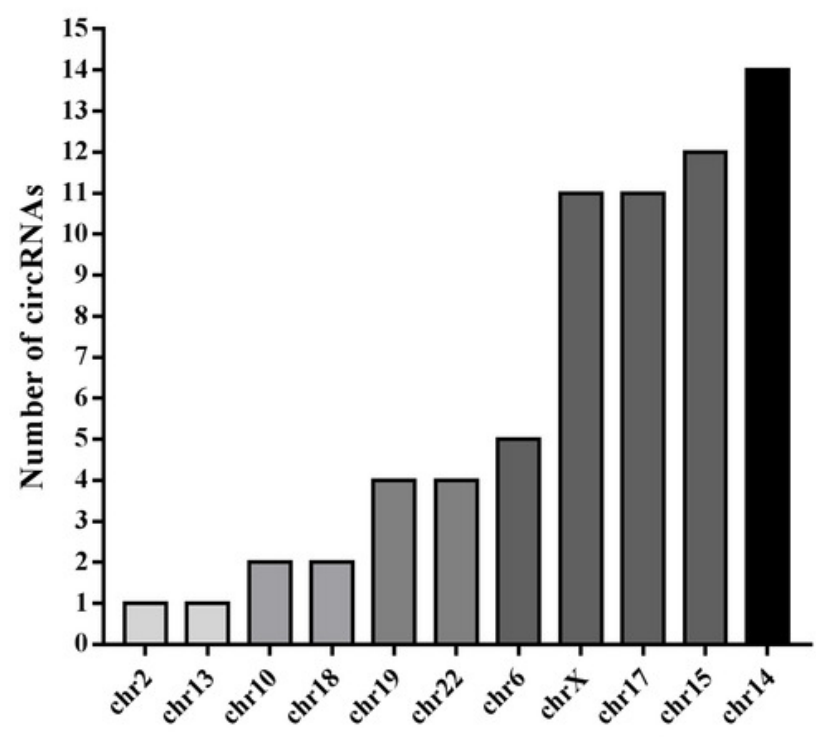

Name of chr

$\mathrm{C}$

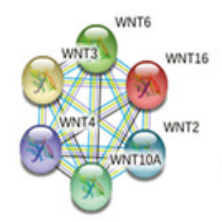

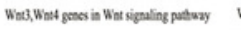

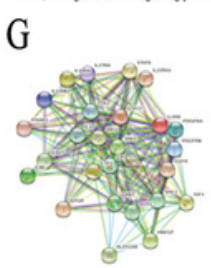

LFF gare in ERK siguling putwory

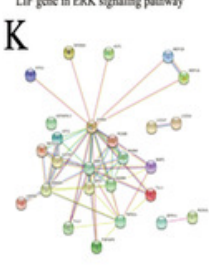

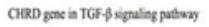

$\mathrm{D}$

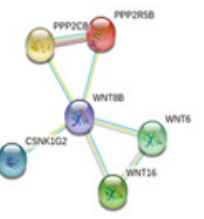

$\mathrm{H}$
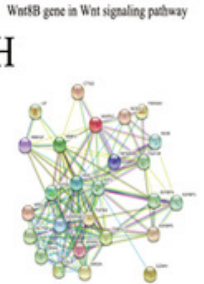

E

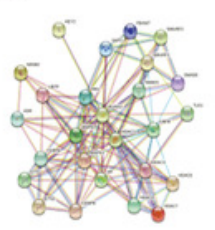

RUNX2 gene in Notch sipaling puthary

I

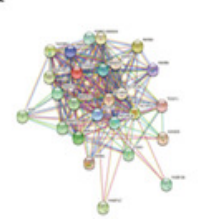

$\mathrm{L}$

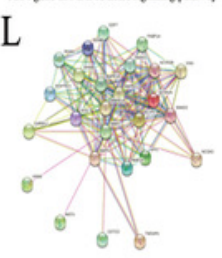

$\mathrm{J}$
B
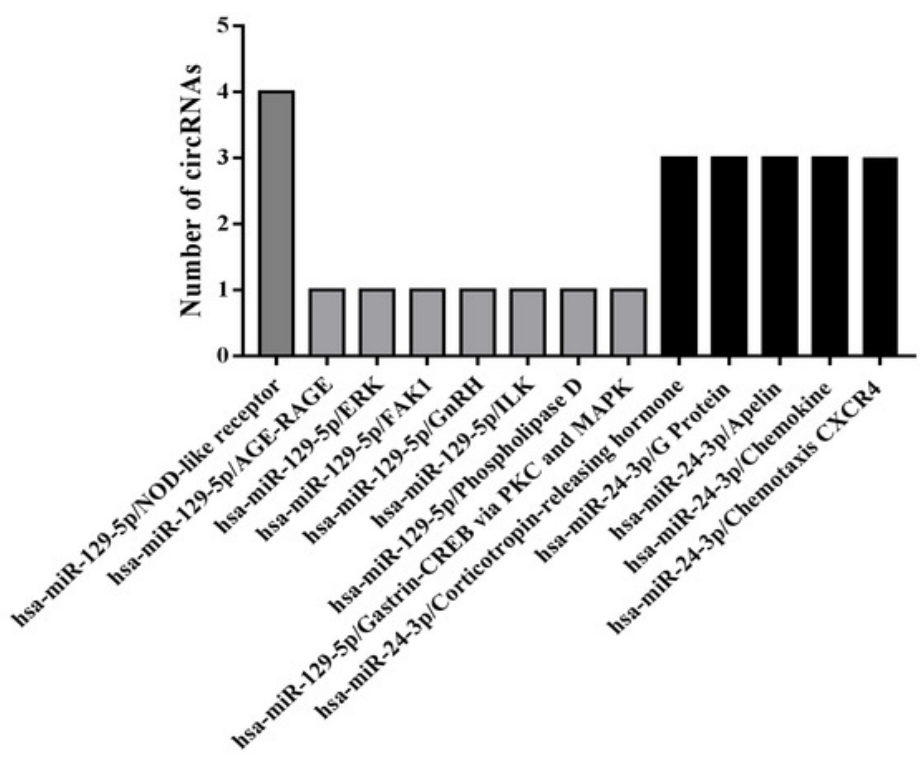

Name of miRNA/signaling pathway

F $\quad$ M
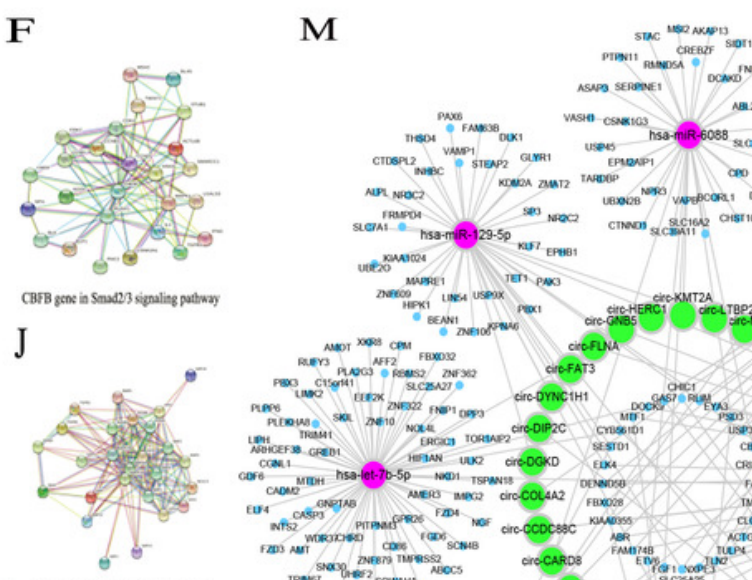

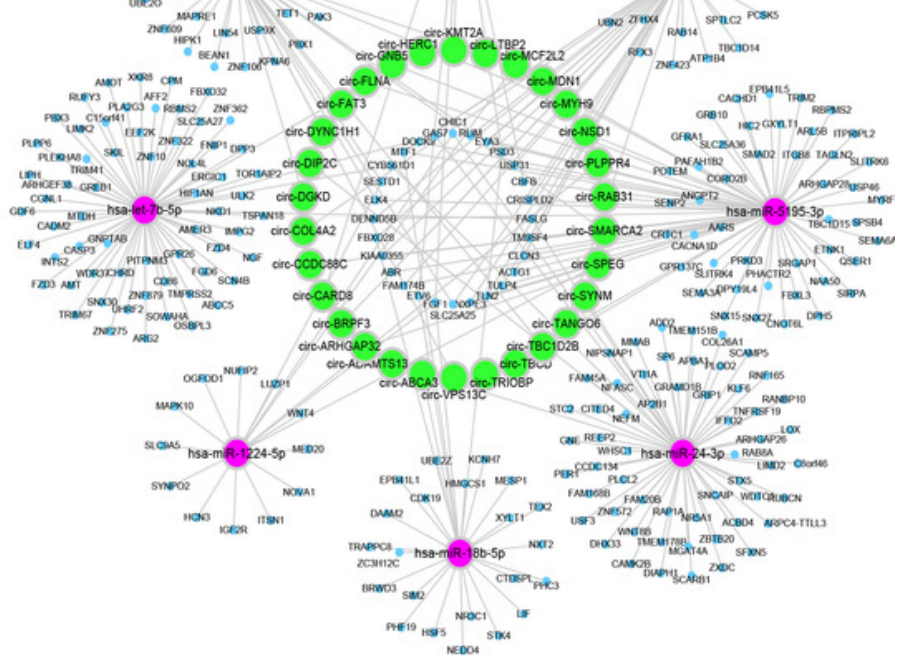


Figure 5

QRT-PCR results of the three differentially expressed circRNAs.

*: The SMAT group compared with the annealed group, FC $>2.0$. (mean $\pm S D, n=3$, * indicates $p<0.05)$.

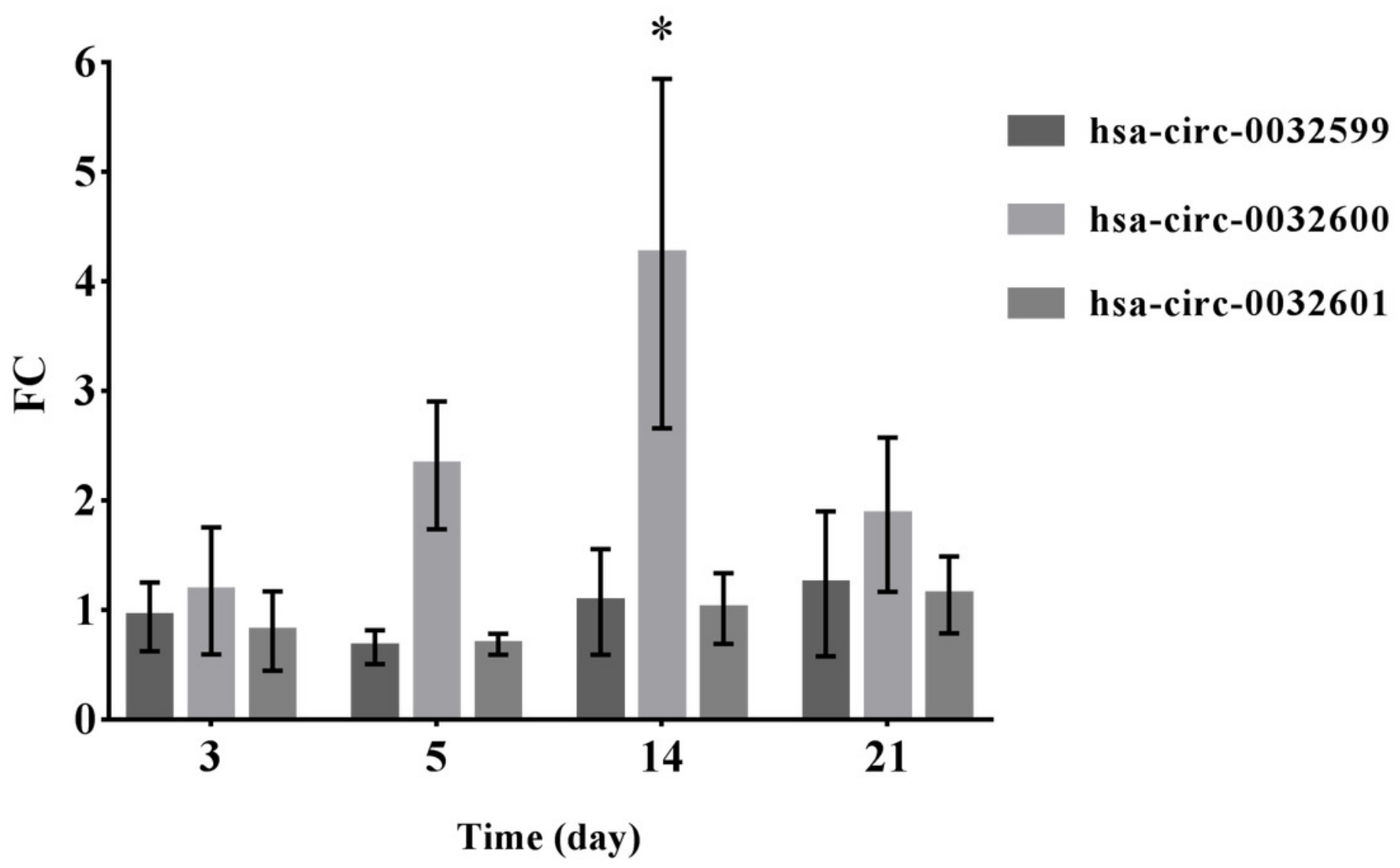

